among the difficulties confronting them may be mentioned that of the energy development of the diffuse giants which stand off the main sequence in such a manner that their energy source cannot easily be reconciled with that of other stars. Explanations have been attempted, but are not entirely satisfactory; and it is suggested that a solution may ultimately be found connected with an important factor-the true distribution of the stars in space.

It is well known that the bright main-sequence stars lie close to the plane of the galaxy, while the diffuse red giants are scattered much more uniformly, and also that the former are conspicuously absent from globular clusters. Some five years ago, Baade found that certain nearby galaxies show similar characteristics, and it is suggested that rotation may be connected with these factors. Many bright stars are rotating rapidly and also revolving with high speed round the centre of the galaxy-unlike the red giants and the globular clusters-and rotation may have an important influence on energy generation. It seems possible that a star which has very little axial rotation could condense in its central regions to an extent that would be difficult if it were rotating rapidly. Although it is probable that such a dense core would involve a temperature sufficiently high for the Bethe cycle to operate, it seems unlikely that the Bethe cycle would lead to stars of this nature as well as to main-sequence stars. Even if the core were sufficiently dense for its gravitational energy to suffice for a long-term energy supply, it is admitted that a very high density would be required.

One serious problem that seems almost intractable is the inadequacy of the 0.008 mass-excess of the hydrogen atom to keep a bright star shining for a long period even on the assumption that it started as practically pure hydrogen and is now nearly pure helium. No star which is 125 times as bright as the sun, and which fits the mass-luminosity relation, can live at this rate as long as the sun has lived, assuming that synthesis is its only source of energy; and it is difficult to account for those stars which have 100,000 times the luminosity of the sun and only 100 times its mass. Either such stars must be very young-a most improbable supposition - or they have become bright in recent times; but even then they would have very little hydrogen left.

Towards the end of the Lecture, reference is made to the work of Bondi and Gold (Mon. Not. Roy. Astro. Soc., 108, 262; 1948) which suggests the continuous generation of matter out of nothing. Assuming that such matter is hydrogen, it would assist in explaining stellar energy provided it could be collected by the stars; but it is very improbable that this could be done with sufficient rapidity. In addition, the hydrogen accretions should penetrate the deep interiors of the stars, and it is difficult to see how this can be done, because the heat produced by impact would stop the motion downwards. Another point which arises is the retardation of rotation by the collection of diffuse extraneous matter; but, as already pointed out, the bright stars are rotating rapidly. One way of surmounting the difficulty-although the General Theory of Relativity does not suggest it-is to restrict this spontaneous generation of matter to places where there is a large amount of matter-the interiors of massive stars. If, however, the diffuse giants are to be included in this scheme, it may be necessary to postulate that the process cannot occur in nonrotating stars.

One tentative suggestion is made. If a very massive star continued to increase more and more, the final result would be that things would get out of control and an explosion would occur which would not result in a mere supernova but in a now expanding universe. The star would disintegrate into portions, each of which would become a new galaxy, and as new supplies of uranium and other radioactive elements would be produced at the same time, the universe resulting from the explosion might show signs of having started at a definite date. It is admitted, however, that the concentration of the generation of matter in the interiors of stars must be regarded as a mere ad hoc speculation.

\section{ASTRONOMY AND NAVIGATION}

A PAPER by D. H. Sadler on "Astronomy and A Navigation" in a recent number of Occasional Notes of the Royal Astronomical Society (13, 2; September 1949) will be very useful to all who are interested in astronomy as applied to navigation. Part $l$ of the paper gives a short historical sketch of the subject and then provides an account of the relationship between the Royal Astronomical Society and navigation. This part will be of special interest to fellows of the Society, many of whom are probably unaware of the important part that the Society has played in the design of the "Nautical Almanac" and also in the contributions of a few of the fellows to nautical astronomy. Although the number of papers is small, they are very useful, and some of them must be regarded as highly valuable contributions to the subject.

Part 2 deals with modern developments-including radar-which raise the question, "Why the need for further development in astronomical navigation ?". The answer to this question lies partly in the enormous ramifications in methods of navigation that have recently been developed, and navigators to-day must be capable of handling all of them. Hence the astronomer has still important work to do, not only in simplifying the principles but also in simplifying their application; and so navigational almanacs are essential to provide in the simplest manner, and to the necessary accuracy, the positions of those heavenly bodies which are used for navigation. A large portion of Part 2 is devoted to a description of the almanacs produced by various countries, and something is said about a few graphical and instrumental methods devised for the solution of the astronomical spherical triangle. In the last section of Part 2, "Observations", special reference is made to the latest sextants, in which random accelerations due to the motion of aircraft are smoothed out, the observations being automatically integrated over periods of one or two minutes. Unfortunately, the Coriolis acceleration cannot be smoothed out in this way, and special tables are required in this case.

Part 3, "The Future", deals, inter alia, with artificial satellites, about which the public has heard much in recent times. After considering certain problems connected with these bodies, the author sums up the situation in the words, "The almanac maker of the era of these artificial satellites would have his full share of problems-both in celestial mechanics and in tabulation". 